TP Periodica Polytechnica Mechanical Engineering

61(2), pp. 135-139, 2017

https://doi.org/10.3311/PPme.9922

Creative Commons Attribution (i)

RESEARCH ARTICLE

\section{Two Theorems for Computation of Projections of Virtual Displacements and Its Application in Structural Analysis}

\author{
Zhenchao $\mathrm{Su}^{1}$, Yanxia Xue ${ }^{1^{*}}$
}

Received 23 August 2016; accepted after revision 27 November 2016

\begin{abstract}
A theorem for planar case and its generalization for spatial case are proposed to determine the projection of a virtual displacement to the orientation under the case of knowing the projections of a virtual displacement to the given two or three orientations for object systems subject to holonomic and scleronomic constraints. Some lemmas corresponding to the two theorems for special cases are given. Applications to structural static analysis are investigated using the two theorems in this paper. Result reveals that the two theorems and corresponding lemmas are easy to be used, shorten the distance between the principle of virtual displacement and its application, and the relating problems can be solved quickly with them.
\end{abstract}

\section{Keywords}

projection of virtual displacement, principle of virtual displacement, engineering mechanics, structural analysis

\footnotetext{
${ }^{1}$ Department of Civil Engineering, Tan Kah Kee College,

Xiamen University, 363105, Zhangzhou city, Fujian Province, China

*Corresponding author, e-mail: xueyx888@126.com
}

\section{Introduction}

It is well known that the principle of virtual displacement (or virtual work) is a main part in analytical statics, and the important basis for analytical dynamics and structural analysis. This principle provides an excellent tool for people to investigate the equilibrium laws of object systems, and plays a supporting role in classical mechanics.

Generally speaking, some current college textbooks [1-7] for engineering mechanics or structural analysis course often propose two main methods for building relations among virtual displacements of different points for the system with holonomic and scleronomic constraints, ie, analytical method and geometrical method. Using analytical method, one can determine the virtual displacements of interest points (usually points of forces) by taking differentials of their position coordinates, and geometrical methods by introducing the concept of the instant center of rotation of virtual displacements. These methods are very useful in application of the principle of virtual displacement. However, in actual experience, we realize that these methods are sometimes not sufficient for analyzing complex structures. For some difficult problems, such as the examples given in this paper, it is impossible or not sufficient to solve them out if only directly using the results given in these current textbooks. Then, how to shorten the distance between the principle of virtual displacement and its application, and give some feasible approaches to realize, is very important and great significance.

In this study, we will gain an insight into the application of the principle of virtual displacement and present two new theorems on how to build up the relations among the projections to different orientations of the virtual displacement of a point. Several examples are given to illustrate the application of two theorems and corresponding lemmas. The article is organized as follows. In Section 2, a theorem is given to find a projection under knowing the two projections to different orientations of a point for planar systems, and two lemmas for special cases are proposed. In Section 3, a theorem for spatial systems as the planar extending situation is given to find a projection under knowing the three projections to different orientations of a point, and three lemmas for special cases are proposed. 


\section{The first theorem for plane cases}

For a planar object system, if the projections of a virtual displacement $\delta \boldsymbol{r}$ of a certain dot to orientations $\boldsymbol{n}_{1}$ and $\boldsymbol{n}_{2}$ are $\delta r_{1}$ and $\delta r_{2}$ separately, shown in Fig. 1, then the projection of the virtual displacement to orientation $\boldsymbol{n}_{3}$ is

$$
\delta r_{3}=\frac{\delta r_{1} \sin (\theta-\phi)+\delta r_{2} \sin \phi}{\sin \theta},
$$

where, angles $\theta$ and $\varphi$ are angles between $\boldsymbol{n}_{1}$ and $\boldsymbol{n}_{2}, \boldsymbol{n}_{1}$ and $\boldsymbol{n}_{3}$ separately.

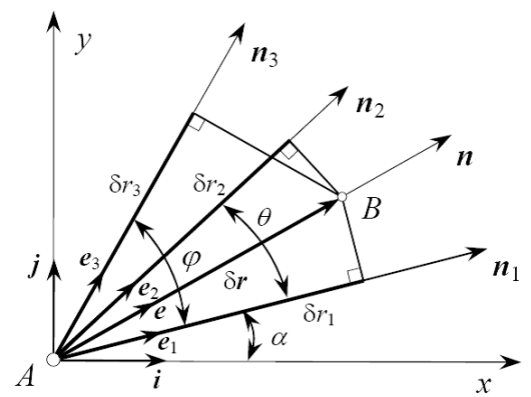

Fig. 1 A virtual displacement and its projections

Proof. Assume $\boldsymbol{e}, \boldsymbol{e}_{1}, \boldsymbol{e}_{2}$ and $\boldsymbol{e}_{3}$ are the unit vectors of the orientations of $\boldsymbol{n}, \boldsymbol{n}_{1}, \boldsymbol{n}_{2}$ and $\boldsymbol{n}_{3}$, separately. Take the start point $A$ of the virtual displacement $\delta \boldsymbol{r}$ as the origin of the coordinate system $x A y$. Let $a$ is the angle between $\boldsymbol{n}_{1}$ and $x$ axis. According to the given conditions, one reads

$$
\begin{aligned}
& \delta \boldsymbol{r} \cdot \boldsymbol{e}_{1}=\delta r_{1}, \\
& \delta \boldsymbol{r} \cdot \boldsymbol{e}_{2}=\delta r_{2} .
\end{aligned}
$$

Hence, one can get

$$
\begin{gathered}
\delta \boldsymbol{r} \cdot(\cos \alpha \boldsymbol{i}+\sin \alpha \boldsymbol{j})=\delta r_{1} \\
\delta \boldsymbol{r} \cdot[\cos (\alpha+\theta) \boldsymbol{i}+\sin (\alpha+\theta) \boldsymbol{j}]=\delta r_{2}
\end{gathered}
$$

where $\boldsymbol{i}, \boldsymbol{j}$ are the standard unit vectors of $x$ and $y$ axis.

By taking the inner products $\delta \boldsymbol{r} \cdot \boldsymbol{i}$ and $\delta \boldsymbol{r} \cdot \boldsymbol{j}$ as unknowns, and solving the equations of (2) and (3), one can get

$$
\begin{gathered}
\delta \boldsymbol{r} \cdot \boldsymbol{i}=\frac{\delta r_{1} \sin (\alpha+\theta)-\delta r_{2} \sin \alpha}{\sin \theta} \\
\delta \boldsymbol{r} \cdot \boldsymbol{j}=\frac{\delta r_{2} \cos \alpha-\delta r_{1} \cos (\alpha+\theta)}{\sin \theta}
\end{gathered}
$$

Then, the projection of the virtual displacement to orientation $\boldsymbol{n}_{3}$ is

$$
\begin{aligned}
\delta r_{3} & =\delta \boldsymbol{r} \cdot \boldsymbol{e}_{3}=\delta \boldsymbol{r} \cdot[\cos (\alpha+\phi) \boldsymbol{i}+\sin (\alpha+\phi) \boldsymbol{j}] \\
& =\cos (\alpha+\phi) \cdot \frac{\delta r_{1} \sin (\alpha+\phi)-\delta r_{2} \sin \alpha}{\sin \theta} \\
& +\sin (\alpha+\phi) \cdot \frac{\delta r_{2} \cos \alpha-\delta r_{1} \cos (\alpha+\phi)}{\sin \theta} \\
& =\frac{\delta r_{1} \sin (\alpha+\phi)+\delta r_{2} \sin \phi}{\sin \theta} .
\end{aligned}
$$

Lemma 1. When $\theta \neq 0\left(180^{\circ}\right), \delta r_{1}=\delta r_{2}=0$, then $\delta r_{3}=0$.

Lemma 2. When $\theta=90^{\circ}$, then $\delta r_{3}=\delta r_{1} \cos \varphi+\delta r_{2} \sin \varphi$.

For demonstrating the effectiveness of this theorem in solving the balancing problems of planar object systems, two examples are given below.

Example 1. Determine the force in member $C D$ of the truss, shown in Fig. 2. Assume all members are pin connected, and $A D=A E=E D=E C=C G=D G=D B=B G=a$.

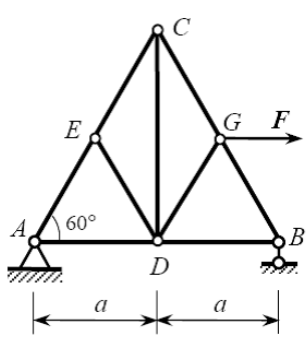

(a)

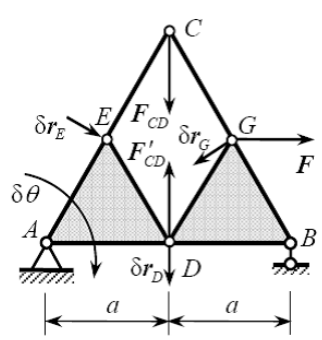

(b)
Fig. 2 Example 1

Solution. To calculate the force in rod $C D$, it is isolated from the system in Fig. 2 (b). Then, the system in Fig. 2 (b) is a mechanism. Obviously, equilateral triangles $A E D$ and $D B G$ can be regarded as rigid plates, and $\triangle A E D$ can be assumed to rotate about $A$ point with $\delta \theta$. Then, $\delta r_{E}=\delta r_{D}=a \delta \theta$. Considering the orientation of $\delta \boldsymbol{r}_{D}$ and character of support $B$, point $B$ is the virtual displacement center of $\triangle D B G$, therefore $\delta r_{G}=\delta r_{D}=a \delta \theta$. Based on the principle of virtual displacement, one reads

$$
\boldsymbol{F} \cdot \delta \boldsymbol{r}_{G}+\boldsymbol{F}_{C D}^{\prime} \cdot \delta \boldsymbol{r}_{D}+\boldsymbol{F}_{C D} \cdot \delta \boldsymbol{r}_{C}=0
$$

Based on the theorem of projection of virtual displacement (i.e. the projections of the virtual displacements of the points from a straight line belonging to a body, on that line, are equal.), the projections of $\delta \boldsymbol{r}_{C}$ to the orientations $E \rightarrow C$ and $G \rightarrow C$ are zeros. Then, by employing the lemma 1 of the above theorem, $\delta \boldsymbol{r}_{C}$ must be zero. Therefore, based on the above analysis and (6), one can get

$$
-F \cdot a \delta \theta \cdot \cos 30^{\circ}-F_{C D}^{\prime} \cdot a \delta \theta=0 .
$$

Because of $\theta \neq 0$, then

$$
F_{C D}^{\prime}=F_{C D}=-F \cdot \cos 30^{\circ}=-\frac{\sqrt{3}}{2} F .
$$

(bar $C D$ in compression).

Example 2. Determine the force in member $D G$ of the truss, shown in Fig. 3. Assume all members are pin connected. 


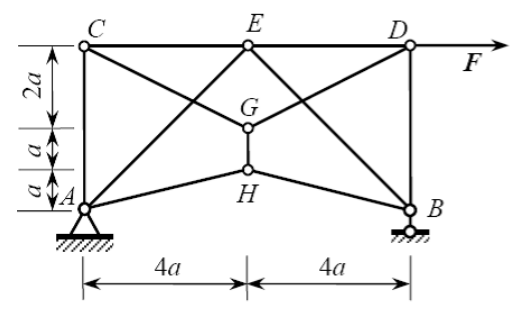

(a)

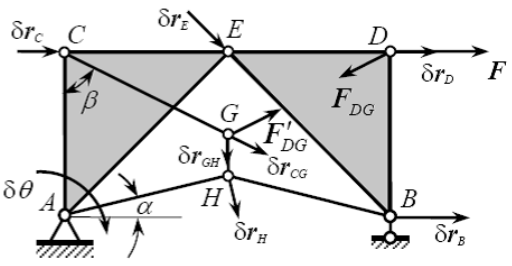

(b)

Fig. 3 Example 2

Solution. To calculate the force in $\operatorname{rod} D G$, it is isolated from the system in Fig. 3 (b). Obviously, triangles $A C E$ and $D B E$ can be regarded as rigid plates, and $\triangle A C E$ can be assumed to rotate about $A$ point with $\delta \theta$. Then, $\delta r_{C}=4 a \delta \theta, \delta r_{E}=4 \sqrt{2} a \delta \theta$. Considering that the orientation of $\delta \boldsymbol{r}_{B}$ is horizontal, the virtual displacement $\delta \boldsymbol{r}_{D}$ must be horizontal, and

$$
\delta r_{D}=\delta r_{E} \cdot \cos 45^{\circ}=4 a \delta \theta
$$

$$
\delta r_{B} \cdot \cos 45^{\circ}=\delta r_{E}, \delta r_{B}=8 a \delta \theta
$$

For the bar $B H$, it is easy to get that

$$
\begin{gathered}
\delta r_{H} \cdot \cos \left(90^{\circ}-2 \alpha\right)=\delta r_{B} \cdot \cos \alpha \\
\delta r_{H}=\frac{1}{2 \sin \alpha} \delta r_{B}=\frac{4 a}{\sin \alpha} \delta \theta .
\end{gathered}
$$

Then, for the bar $G H$, the projection of $\delta \boldsymbol{r}_{G}$ to the orientation $G \rightarrow H$ is

$$
\delta r_{G H}=\delta r_{H} \cdot \cos \alpha=\frac{4 a \cos \alpha}{\sin \alpha} \delta \theta .
$$

For the bar $C G$, the projection of $\delta \boldsymbol{r}_{G}$ to the orientation $C \rightarrow G$ is

$$
\delta r_{C G}=\delta r_{C} \cdot \cos \left(90^{\circ}-\beta\right)=4 a \sin \beta \delta \theta
$$

For the point $G$, the projections $\delta r_{G H}$ and $\delta r_{C G}$ are known. By employing the formula (1) of the above theorem, the projection of $\delta \boldsymbol{r}_{G}$ to the orientation $G \rightarrow D$ is

$$
\begin{aligned}
\delta r_{G D} & =\frac{\delta r_{G H} \sin \left\{\beta-\left[\beta+2\left(90^{\circ}-\beta\right)\right]\right\}+\delta r_{C G} \sin \left[\beta+2\left(90^{\circ}-\beta\right)\right]}{\sin \beta} \\
& =\left[\frac{-4 a \cos \alpha \sin 2 \beta}{\sin \alpha \sin \beta}+4 a \sin \beta\right] \delta \theta \\
& =(-8 a \cot \alpha \cos \beta+4 a \sin \beta) \delta \theta \\
& =\left(-8 a \cdot 4 \cdot \frac{2}{2 \sqrt{5}}+4 a \cdot \frac{4}{2 \sqrt{5}}\right) \delta \theta=-\frac{24}{\sqrt{5}} a \delta \theta .
\end{aligned}
$$

By employing the principle of virtual displacement, one can get

$$
\boldsymbol{F} \cdot \delta \boldsymbol{r}_{D}+\boldsymbol{F}_{D G} \cdot \delta \boldsymbol{r}_{D}+\boldsymbol{F}_{D G}^{\prime} \cdot \delta \boldsymbol{r}_{G}=0
$$

Therefore, based on the above analysis and (7), one can get

$$
F \cdot 4 a \delta \theta-F_{D G} \cdot 4 a \delta \theta \cdot \cos \left(90^{\circ}-\beta\right)-F_{D G}^{\prime} \cdot \frac{24}{\sqrt{5}} a \delta \theta=0
$$

Because $\delta \theta \neq 0, F_{D G}=F_{D G}^{\prime}$, then

$$
F_{D G}=\frac{F}{\sin \beta+\frac{6}{\sqrt{5}}}=\frac{F}{\frac{2}{\sqrt{5}}+\frac{6}{\sqrt{5}}}=\frac{\sqrt{5} F}{8}=0.279 F .
$$

\section{The second theorem for spatial cases}

If the projections of the virtual displacement $\delta \boldsymbol{r}$ of a dot to three non-coplanar orientations $\boldsymbol{n}_{1}, \boldsymbol{n}_{2}, \boldsymbol{n}_{3}$ are $\delta r_{1}, \delta r_{2}$, $\delta r_{3}$, then the projection $\delta r_{4}$ of the virtual displacement to the orientation $\boldsymbol{n}_{4}$ is

$$
\delta r_{4}=\left(e_{4 x}, e_{4 y}, e_{4 z}\right)\left[\begin{array}{lll}
e_{1 x} & e_{1 y} & e_{1 z} \\
e_{2 x} & e_{2 y} & e_{2 z} \\
e_{3 x} & e_{3 y} & e_{3 z}
\end{array}\right]^{-1}\left(\begin{array}{c}
\delta r_{1} \\
\delta r_{2} \\
\delta r_{3}
\end{array}\right)
$$

where $e_{i x}, e_{i z}, e_{i z}$ are projections of unit vector $\boldsymbol{e}_{i}$ of spatial orientation $\boldsymbol{n}_{i}$ to the Cartesian coordinate axes $x, y, z$, separately. $(i=1,2,3,4)$

Proof. Assuming that $\boldsymbol{i}, \boldsymbol{j}, \boldsymbol{k}$ are the standard unit orthogonal vectors of the spatial coordinate system, based on the given conditions, one reads

$$
\boldsymbol{e}_{i}=e_{i x} \boldsymbol{i}+e_{i y} \boldsymbol{j}+e_{i z} \boldsymbol{k}, \quad i=1,2,3,4
$$

and

$$
\left\{\begin{array}{l}
e_{1 x} \delta \boldsymbol{r} \cdot \boldsymbol{i}+e_{1 y} \delta \boldsymbol{r} \cdot \boldsymbol{j}+e_{1 z} \delta \boldsymbol{r} \cdot \boldsymbol{k}=\delta r_{1} \\
e_{2 x} \delta \boldsymbol{r} \cdot \boldsymbol{i}+e_{2 y} \delta \boldsymbol{r} \cdot \boldsymbol{j}+e_{2 z} \delta \boldsymbol{r} \cdot \boldsymbol{k}=\delta r_{2} \\
e_{3 x} \delta \boldsymbol{r} \cdot \boldsymbol{i}+e_{3 y} \delta \boldsymbol{r} \cdot \boldsymbol{j}+e_{3 z} \delta \boldsymbol{r} \cdot \boldsymbol{k}=\delta r_{3}
\end{array}\right.
$$

or

$$
\left[\begin{array}{lll}
e_{1 x} & e_{1 y} & e_{1 z} \\
e_{2 x} & e_{2 y} & e_{2 z} \\
e_{3 x} & e_{3 y} & e_{3 z}
\end{array}\right]\left(\begin{array}{l}
\delta \boldsymbol{r} \cdot \boldsymbol{i} \\
\delta \boldsymbol{r} \cdot \boldsymbol{j} \\
\delta \boldsymbol{r} \cdot \boldsymbol{k}
\end{array}\right)=\left(\begin{array}{c}
\delta r_{1} \\
\delta r_{2} \\
\delta r_{3}
\end{array}\right)
$$

Because the three orientations $\boldsymbol{e}_{1}, \boldsymbol{e}_{2}, \boldsymbol{e}_{3}$ are non-coplanar, the coefficient matrix is reversible. Hence, one can get

$$
\left(\begin{array}{c}
\delta \boldsymbol{r} \cdot \boldsymbol{i} \\
\delta \boldsymbol{r} \cdot \boldsymbol{j} \\
\delta \boldsymbol{r} \cdot \boldsymbol{k}
\end{array}\right)=\left[\begin{array}{lll}
e_{1 x} & e_{1 y} & e_{1 z} \\
e_{2 x} & e_{2 y} & e_{2 z} \\
e_{3 x} & e_{3 y} & e_{3 z}
\end{array}\right]^{-1}\left(\begin{array}{c}
\delta r_{1} \\
\delta r_{2} \\
\delta r_{3}
\end{array}\right)
$$


Thus, the projection of the virtual displacement $\delta \boldsymbol{r}$ to orientation $\boldsymbol{n}_{4}$ is

$$
\begin{aligned}
\delta r_{4} & =\left(e_{4 x}, e_{4 y}, e_{4 z}\right)\left(\begin{array}{c}
\delta \boldsymbol{r} \cdot \boldsymbol{i} \\
\delta \boldsymbol{r} \cdot \boldsymbol{j} \\
\delta \boldsymbol{r} \cdot \boldsymbol{k}
\end{array}\right) \\
& =\left(e_{4 x}, e_{4 y}, e_{4 z}\right)\left[\begin{array}{lll}
e_{1 x} & e_{1 y} & e_{1 z} \\
e_{2 x} & e_{2 y} & e_{2 z} \\
e_{3 x} & e_{3 y} & e_{3 z}
\end{array}\right]^{-1}\left(\begin{array}{l}
\delta r_{1} \\
\delta r_{2} \\
\delta r_{3}
\end{array}\right) .
\end{aligned}
$$

Lemma 3. If the unit vectors $\boldsymbol{e}_{i}(i=1,2,3,4)$ are normal orthogonal unit vectors, the projection $\delta r_{4}$ of the virtual displacement to the orientation $\boldsymbol{n}_{4}$ is

$$
\delta r_{4}=\left(e_{4 x}, e_{4 y}, e_{4 z}\right)\left[\begin{array}{lll}
e_{1 x} & e_{2 x} & e_{3 x} \\
e_{1 y} & e_{2 y} & e_{3 y} \\
e_{1 z} & e_{2 z} & e_{3 z}
\end{array}\right]\left(\begin{array}{c}
\delta r_{1} \\
\delta r_{2} \\
\delta r_{3}
\end{array}\right)
$$

Lemma 4. If the unit vectors $\boldsymbol{e}_{i}$ of orientations $\boldsymbol{n}_{i}(i=1,2,3,4)$ are non-planar vectors, and the projections of the virtual displacement of a dot to $\boldsymbol{n}_{i}$ are all zeros, then the virtual displacement must be zero vector, and projection of the virtual displacement to $\boldsymbol{n}_{4}$ (every orientation) must be zero.

Lemma 5. If the unit vectors $\boldsymbol{e}_{i}(i=1,2,3,4)$ are standard unit orthogonal vectors of the Cartesian coordinate axes $x, y$, $z$, separately, the projection of the virtual displacement to $\boldsymbol{e}_{i}$ is $\delta r_{i}$, then the projection of the virtual displacement to the orientation $\boldsymbol{n}_{4}$ is

$$
\delta r_{4}=e_{4 x} \delta r_{1}+e_{4 y} \delta r_{2}+e_{4 z} \delta r_{3}
$$

Example 3. Determine the force in member $A C$ of the spatial truss, shown in Fig. 4 (a). Assume all members are pin connected. $F_{1}=5 \mathrm{kN}, F_{2}=4 \mathrm{kN}, F_{3}=2 \mathrm{kN}, F_{4}=1.5 \mathrm{kN}, F_{5}=1.5 \mathrm{kN}$.

Solution. To calculate the force in $\operatorname{rod} A C$, it is isolated from the system in Fig. 4 (b). Obviously, hinge points $E, D, C$ and $B$ are fixed. Therefore, the projection of point $A$ to the orientations $B \rightarrow A$ and $D \rightarrow A$ are zeros. Assuming that the projection along the $\boldsymbol{F}_{4}$ direction is $\delta r$, considering that the unit vectors of the direction $B \rightarrow A, A \rightarrow C, D \rightarrow A$ and $\boldsymbol{F}_{4}$ are as follows

$$
\begin{gathered}
\boldsymbol{e}_{B A}=\left(\frac{4}{5},-\frac{3}{5}, 0\right), \quad \boldsymbol{e}_{A C}=(-1,0,0), \\
\boldsymbol{e}_{D A}=(0,0,1), \quad \boldsymbol{e}_{F_{4}}=(0,-1,0)
\end{gathered}
$$

Then, utilizing the second theorem for spatial case, the projection along the $A \rightarrow C$ direction is

$$
\begin{aligned}
\delta r_{4} & =(-1,0,0)\left[\begin{array}{ccc}
\frac{4}{5} & -\frac{3}{5} & 0 \\
0 & 0 & 1 \\
0 & -1 & 0
\end{array}\right]^{-1}\left(\begin{array}{c}
0 \\
0 \\
\delta r
\end{array}\right) \\
& =(-1,0,0)\left[\begin{array}{ccc}
\frac{5}{4} & 0 & -\frac{3}{4} \\
0 & 0 & -1 \\
0 & 1 & 0
\end{array}\right]\left(\begin{array}{c}
0 \\
0 \\
\delta r
\end{array}\right)=\frac{3}{4} \delta r .
\end{aligned}
$$

By employing the principle of virtual displacement, one reads

$$
\boldsymbol{F}_{A C} \cdot \delta \boldsymbol{r}_{A}+\boldsymbol{F}_{4} \cdot \delta \boldsymbol{r}_{A}=0
$$

Based on the above analysis and (11), one can get

$$
F_{A C} \cdot \frac{3}{4} \delta r+F_{4} \cdot \delta r=0
$$

Because $\delta \theta \neq 0$, then one can get

$$
F_{A C}=-\frac{4}{3} F_{4}=-\frac{4}{3} \times 1.5 \mathrm{kN}=-2.0 \mathrm{kN} \text {. }
$$

a)
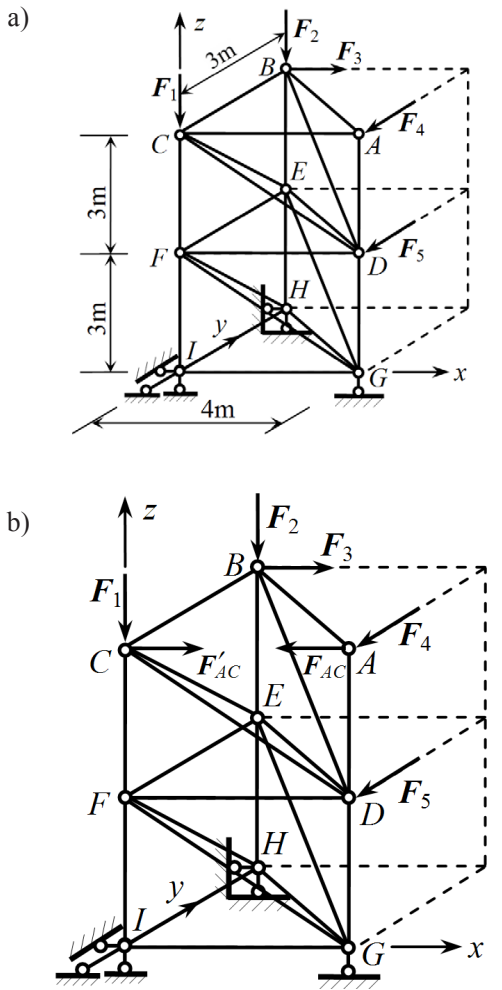

Fig. 4 Example 3 


\section{Conclusions}

This paper mainly faces to the difficulty of computation of virtual work, investigates on the projection of a dot's virtual displacement to a given orientation, propose two theorems and corresponding lemmas, and discuss their application for analyzing the forces of structural members. Computation procession reveals that the formula for planar situation is easy to use, and the formula for spatial situation is normalized and easy to be remembered. Combining with current methods, these results will be very helpful to analyze inner forces of complicit structures. It's necessary to say that the two theorems can be used in dynamics when dynamical problems convert into static problems in form by utilizing D' Alembert's principle.

\section{Acknowledgement}

This paper is supported by the $13^{\text {th }}$ five-year educational scientific research planning programs in Fujian (Grant No. FJJKCGZ16-152). The authors gratefully acknowledge its financial supports.

\section{References}

[1] Hibbeler, R. C. "Engineering Mechanics: Statics." 12th edition. Prentice Hall, 2010

[2] Pytel, A., Kiusalaas, J., Sharma, I. "Engineering Mechanics: Statics." 3rd edition, SI, CENGAGE Learning, 2010.

[3] Bedford, A., Fowler, W. "Engineering Mechanics: Statics." 5th edition, Pearson Education Inc., 2008.

[4] Gross, D., Hauger, W., Schröder, J., Wall, W. A., Rajapakse, N. "Engineering Mechanics 1: Statics." Springer-Verlag Berlin Heidelberg, 2009.

[5] Kumar, K. L. "Engineering Mechanics." 3rd edition, McGraw-Hill Publishing Company Limited, 2003.

[6] Jong, I. C., Rogers, B. G. "Engineering Mechanics: Statics." Saunders College Publishing, 1991.

[7] Ranzi, G., Gilbert, R. I. "Structural Analysis: Principles, Methods and Modelling." CRC Press, 2015. 